
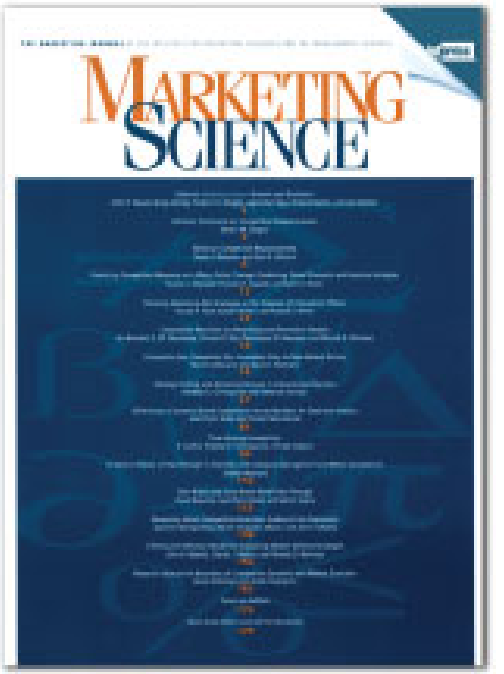

\title{
Marketing Science
}

Publication details, including instructions for authors and subscription information: http:// pubsonline.informs.org

\section{To Share or Not to Share: Demand Forecast Sharing in a Distribution Channel}

Baojun J iang, Lin Tian, Yifan Xu, Fuqiang Zhang

\section{To cite this article:}

Baoj un J iang, Lin Tian, Yifan Xu, Fuqiang Zhang (2016) To Share or Not to Share: Demand Forecast Sharing in a Distribution Channel. Marketing Science 35(5):800-809. http:// dx. doi. org/ 10.1287/ mksc. 2016. 0981

\section{Full terms and conditions of use: http://pubsonline.informs.org/page/terms-and-conditions}

This article may be used only for the purposes of research, teaching, and/or private study. Commercial use or systematic downloading (by robots or other automatic processes) is prohibited without explicit Publisher approval, unless otherwise noted. For more information, contact permissions@informs.org.

The Publisher does not warrant or guarantee the article's accuracy, completeness, merchantability, fitness for a particular purpose, or non-infringement. Descriptions of, or references to, products or publications, or inclusion of an advertisement in this article, neither constitutes nor implies a guarantee, endorsement, or support of claims made of that product, publication, or service.

Copyright (c) 2016, INFORMS

Please scroll down for article-it is on subsequent pages

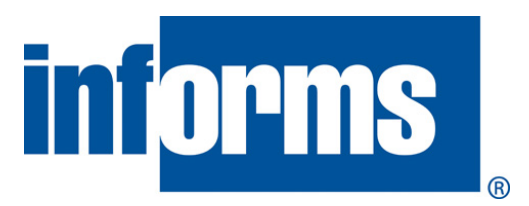

INFORMS is the largest professional society in the world for professionals in the fields of operations research, management science, and analytics.

For more information on INFORMS, its publications, membership, or meetings visit http:// www. informs.org 


\title{
To Share or Not to Share: Demand Forecast Sharing in a Distribution Channel
}

\author{
Baojun Jiang \\ Olin Business School, Washington University in St. Louis, St. Louis, Missouri 63130, baojunjiang@wustl.edu \\ Lin Tian \\ School of International Business Administration, Shanghai University of Finance and Economics, 200433 Shanghai, China, \\ tian.lin@mail.shufe.edu.cn \\ Yifan $\mathrm{Xu}$ \\ School of Management, Fudan University, 200433 Shanghai, China, yfxu@fudan.edu.cn \\ Fuqiang Zhang \\ Olin Business School, Washington University in St. Louis, St. Louis, Missouri 63130, fzhang22@wustl.edu
}

\begin{abstract}
$\mathrm{T}_{\mathrm{d} m \mathrm{~m}}^{\mathrm{h}}$ his paper studies information sharing in a distribution channel where the manufacturer possesses better demand-forecast information than the downstream retailer. We examine three information-sharing formats: no information sharing (i.e., the manufacturer ex ante commits to not sharing its forecast), voluntary information sharing (i.e., the manufacturer makes the sharing decision ex post after receiving the forecast), and mandatory information sharing (i.e., the manufacturer is mandated to share its forecast). We characterize the equilibrium outcomes under the three sharing formats and investigate the firms' preferences regarding these formats. It is shown that when the retailer is risk-neutral, both firms are indifferent between voluntary and mandatory sharing. Among the three formats, ex ante, the retailer prefers the no-sharing format whereas the manufacturer prefers the mandatory-sharing format. In addition, we find that a more accurate forecast benefits both firms under voluntaryand mandatory-sharing formats, but may hurt both firms under the no-sharing format. Finally, we show that risk aversion plays a critical role in the firms' sharing decisions and the impact of forecast accuracy. Specifically, when the retailer is risk-averse, the manufacturer may prefer the no-sharing format over the voluntary-sharing format, and improving forecast accuracy may hurt both firms even under voluntary sharing.
\end{abstract}

Keywords: information sharing; signaling; forecast accuracy; risk aversion; distribution channel

History: Received: November 6, 2013; accepted: November 28, 2015; Preyas Desai served as the editor-in-chief and

Miguel Villas-Boas served as associate editor for this article. Published online in Articles in Advance May 13, 2016.

\section{Introduction}

Firms often face highly uncertain demand when launching new products or expanding into new markets. This creates a challenge because firms have to make marketing and operations decisions before such uncertainty is resolved. In industries characterized by notoriously volatile demand (e.g., fashion, high technology, and seasonal goods), manufacturers tend to invest heavily to obtain better forecasts of market demand for their new products. For example, Sport Obermeyer adopted the Accurate Response program that equipped the company with superior demand forecasting and production-planning capabilities (Fisher et al. 1994). Zara, a fashion company, developed a sophisticated system to forecast demand for thousands of new designs each year to guide both company-operated shops and franchised stores. The availability of advanced information technology and data analytics tools today allows firms to more effectively collect, analyze, and act on information from various sources. Large brand-owning manufacturers such as Procter \& Gamble (P\&G) often conduct extensive market research on consumer preferences and demand when they design and market their new products. For new products or products with short life cycles, retailers may have to rely on the upstream manufacturers for market information (Mohr and Sohi 1995, Agency Sales 2003). The question is: Should the manufacturer share its demand forecast with the downstream retailer and, if so, using what sharing format?

We study three information-sharing formats. The first format is "no information sharing," which arises if either the manufacturer ex ante commits to not sharing the forecast (e.g., the manufacturer has a strict dataconfidentiality policy) or the retailer does not make the upfront investment (in technology or human resources) necessary for information sharing. The second is "voluntary information sharing," where the manufacturer decides whether to share after receiving the forecast and the retailer will adjust its pricing strategy accordingly. 
The third format is "mandatory information sharing," i.e., the manufacturer always shares the forecast; for example, a third-party agency may be contracted to forecast demand and send the report to both channel members, or the channel members may ex ante set up an electronic data interchange (EDI) or other information platforms to automatically share forecast data (e.g., Nabisco, P\&G; see Voluntary Interindustry Commerce Solutions Association (VISCA) 1999).

What are the manufacturer's and the retailer's preferences as to the three sharing formats? One might intuit that the manufacturer will prefer voluntary sharing and the retailer will prefer mandatory sharing. We show that this may not be true when both the channel members' strategic pricing behaviors are taken into account. Furthermore, forecast accuracy is important in uncertain markets and may depend on the firm's proximity to and past interactions with its targeted customers, and the availability and quality of its customer databases and market research tools and experience (He et al. 2008). Question: Will forecast accuracy improvement benefit the firms, and how does it affect the firms' preferences over sharing formats? Demand uncertainty also raises another salient and important issue, i.e., channel members' risk attitude. Though national brand manufacturers such as $P \& G$ and Kellogg are likely risk-neutral, many smaller-scale retailers (e.g., Aldi and Schnucks) are probably more risk-averse. We will analyze how risk aversion influences pricing decisions and information-sharing preferences in the channel.

Our research contributes to the extant literature on information sharing in distribution channels (e.g., Chu and Messinger 1997, Cachon and Fisher 2000, Lee et al. 2000, Aviv 2001, Li 2002, Li and Zhang 2008, Guo 2009). These papers primarily assume that downstream retailers have better information. By contrast, He et al. (2008) show that when both the manufacturer and the retailer have private demand information, information sharing might not improve channel profits. Yet they ignore the possibility that, when information is not shared, the retailer may make rational inferences about the manufacturer's private information from its wholesale price. Dukes et al. (2011) show that with such inferences, bilateral information sharing can benefit only the manufacturer at the expense of the retailer and consumers. Gal-Or et al. (2008) show that when one manufacturer and two competing retailers all have private information but the manufacturer's information is more accurate, the manufacturer may share its information only with the less-informed retailer.

Our paper differs from these aforementioned papers in three ways. First, in our paper, only the upstream manufacturer possesses private demand forecast, not the retailer. This setting is practical and important for new products. Second, we also study the case that the manufacturer can decide whether to share after obtaining the forecast rather than only before obtaining it as in the aforementioned papers. In these aspects, the Guo and Iyer (2010) paper is similar to our paper, but like He et al. (2008), they also assume that the retailer makes no rational inferences about the manufacturer's demand information from its wholesale price, whereas such inferences are a key aspect of our analysis. Third, we study the impact of forecast accuracy and the retailer's risk aversion on pricing and information sharing.

Our research complements the literature that studies the impact of information accuracy. Iyer et al. (2007) examine the trade-offs between demand information and inventory; they find that with mandatory sharing, the manufacturer may not prefer more accurate information. Miyaoka and Hausman (2008) show that when the downstream firm has private demand forecast and the upstream firm makes a capacity decision, a perfectly accurate forecast may lead to lower channel profits than a completely uninformative forecast. Taylor and Xiao (2010) show that both the manufacturer and the retailer may be worse off if the retailer's forecast accuracy improves under a wholesale price contract. Shin and Tunca (2010) show that, in a channel with one manufacturer and multiple competing resellers, the retailer may not make accuracy-improving investments. Unlike our paper, all these studies (except Iyer et al. 2007) assume that the retailer, rather than the upstream manufacturer, has better forecast information. They do not compare the three possible information-sharing formats. They also do not study the effect of risk aversion.

We also contribute to the literature on signaling in various problem settings, including quality signaling using money-back guarantees (Moorthy and Srinivasan 1995), signaling of price images (Simester 1995, Shin 2005), demand or cost information signaling using two-part tariffs (Tirole 1988, Gallini and Wright 1990), cost signaling to inequity-averse consumers (Guo and Jiang 2016) or to forward-looking consumers in a dynamic setting (Jiang et al. 2016), signaling by ethical service providers (Jiang et al. 2014), a seller's demand signaling to the platform owner (Jiang et al. 2011), a manufacturer's demand signaling to retailers using multiple messages (Desai 2000), and a franchisor's demand signaling using two- and three-part tariffs to an uninformed franchisee whose unobservable effort also influences demand (Desai and Srinivasan 1995). By contrast, our paper focuses on demand signaling with the commonly used wholesale price contract and studies the impact of forecast accuracy and risk aversion under different information-sharing contexts.

We highlight our key findings below. Our base model with a risk-neutral retailer shows that, first, voluntary sharing is equivalent to mandatory sharing, and interestingly, that the manufacturer prefers mandatory sharing to no sharing and the retailer prefers no 
sharing to mandatory sharing. This is because, under the no-sharing format, the manufacturer must distort its wholesale price downward to convey a low forecast to the retailer to induce an appropriately low retail price; this distortion (i.e., the manufacturer's signaling cost) will benefit the retailer. Second, the impact of forecast accuracy depends on the information-sharing format. A more accurate forecast will benefit both firms under voluntary and mandatory sharing, but may lead to lower profits for both firms under no sharing. The extant literature has shown examples of "accuracy trap," i.e., an improvement in forecast accuracy can lead to lower revenue for the informed party (Laucka 2005). Shin and Tunca (2010) explain this phenomenon with horizontal competition where the downstream retailer has superior demand forecast. By contrast, we show that under the no-sharing format, because of strategic pricing decisions, both the better informed party and the less informed party may suffer from a more accurate forecast even when it is the upstream manufacturer that possesses the superior forecast information.

Our model extension studies the retailer's risk aversion. First, when the retailer is risk-averse, voluntary sharing and mandatory sharing are no longer equivalent; the manufacturer prefers voluntary sharing to mandatory sharing whereas the retailer prefers mandatory sharing to voluntary sharing. This is because a risk-averse retailer is more sensitive to demand uncertainty and can reduce that uncertainty through information sharing, which will lead to a greater retail price increase, benefiting the retailer and hurting the manufacturer. Unlike in the case of a risk-neutral retailer, the manufacturer may prefer not sharing its forecast if the retailer is sufficiently risk-averse. Given that under voluntary sharing the manufacturer can ex post choose whether to share, the retailer can infer more information when the forecast is not shared compared with the (ex ante) no-sharing case. Because risk aversion makes the retailer's strategic pricing more sensitive to reduced demand uncertainty, the manufacturer may prefer no sharing as the retailer's risk aversion increases. Second, by contrast to the case of a risk-neutral retailer, under voluntary sharing, an improvement in forecast accuracy may lead to lower profits for both firms when the retailer is risk-averse. These findings indicate that it is important for the upstream manufacturer to take the retailer's risk aversion into account when making information-sharing and forecast investment decisions.

\section{Model}

Consider a channel with a manufacturer selling its product at a wholesale price $w$ to a retailer, who sells the product to consumers at a retail price $p$. This paper focuses on wholesale price contracts, which are very common in practice and in the existing literature on information sharing; this allows for direct comparisons of our results with those in the literature.

Market Demand. Market demand is $D=\theta(z)-p$, where the total market size, $\theta(z)$, is uncertain and depends on the demand state $z$. For convenience, denote $\theta(z)$ by $\theta_{z}$. Demand can be in a high $(H)$ or low $(L)$ state, with corresponding market sizes of $\theta_{H}=(1+\Delta) \bar{\theta}$ and $\theta_{L}=(1-\Delta) \bar{\theta}$, respectively. These two demand states occur with equal ex ante probabilities: ${ }^{1}$ $\operatorname{Pr}(H)=\operatorname{Pr}(L)=\frac{1}{2}$. Both the manufacturer and the retailer know the prior distribution of $\theta$. The parameter $\bar{\theta}>0$ is the average market size and $\Delta>0$ measures the level of demand uncertainty.

Demand Forecast. The manufacturer can obtain a private forecast (or signal) $s$ about market demand. Similar to the extant literature on information sharing, we study the truthful sharing of forecast (i.e., the manufacturer cannot reveal a different demand signal from the one it actually received). ${ }^{2}$ Such sharing can be done via dataexchange platforms (e.g., EDI and Internet) or through a third-party intermediary or market research agency. The forecast information may include, for example, market research data and reports by the manufacturer or a third-party agent. The signal $s$ has two possible values: $s=h$ and $s=l$, corresponding to the two demand states $H$ and $L$, respectively. Let $\rho \in(0,1)$ be the measure of the accuracy of the signal. When $\rho=1$, the forecast is perfectly reliable: $\operatorname{Pr}(h \mid H)=\operatorname{Pr}(l \mid L)=1$; when $\rho=0$, the forecast is completely unreliable, i.e., the posterior probabilities remain the same as the prior. Following the literature (e.g., Iyer et al. 2007), we assume the manufacturer's forecast to be unbiased such that the unconditional probability of a signal indicating any demand state is equal to the prior probability of that state, i.e., $\operatorname{Pr}(h)=\operatorname{Pr}(H)$ and $\operatorname{Pr}(l)=\operatorname{Pr}(L)$. Note that $\operatorname{Pr}(h)=\operatorname{Pr}(H) \operatorname{Pr}(h \mid H)+\operatorname{Pr}(L) \operatorname{Pr}(h \mid L)$ and $\operatorname{Pr}(l)=$ $\operatorname{Pr}(H) \operatorname{Pr}(l \mid H)+\operatorname{Pr}(L) \operatorname{Pr}(l \mid L)$. Given these properties, one can readily derive $\operatorname{Pr}(h \mid H)=\operatorname{Pr}(l \mid L)=(1+\rho) / 2$ and $\operatorname{Pr}(h \mid L)=\operatorname{Pr}(l \mid H)=(1-\rho) / 2$. Similarly, $\operatorname{Pr}(H \mid h)=$ $\operatorname{Pr}(L \mid l)=(1+\rho) / 2$ and $\operatorname{Pr}(L \mid h)=\operatorname{Pr}(H \mid l)=(1-\rho) / 2$. Note that $\operatorname{Pr}(H \mid h)=\operatorname{Pr}(L \mid l)>\operatorname{Pr}(H)=\operatorname{Pr}(L)$ for any $\rho>0$, which implies that the forecast signal always improves the demand information. As $\rho$ increases, the accuracy of the manufacturer's demand information increases, but as long as $\rho<1$, the manufacturer's forecast is not perfect, i.e., there is a positive probability that the realized demand differs from the forecast.

\footnotetext{
${ }^{1}$ The online appendix (available as supplemental material at http://dx .doi.org/10.1287/mksc.2016.0981) analyzes more general prior beliefs and demand distributions; our main results are qualitatively the same.

${ }^{2}$ That is, our analysis focuses on "hard" information that can be truthfully revealed. Alternatively, the demand forecast could be "soft" information (e.g., managers' personal opinions about future demand) that is subject to manipulation and thus may become cheap talk. We do not consider the sharing of such soft information in this paper.
} 
Sequence of Events. The game proceeds as follows. First, the manufacturer or the retailer chooses from the three information-sharing formats, i.e., no sharing, voluntary sharing, and mandatory sharing. The second stage of the game has several steps. First, the manufacturer observes the forecast signal $s$, and may or may not share $s$ with the retailer depending on the format chosen earlier; second, the manufacturer chooses the wholesale price $w$ and then the retailer sets her retail price $p$; last, market demand and firms' profits are realized.

We normalize the manufacturer's marginal cost to zero; including a positive production cost will not qualitatively change our results. The manufacturer and the retailer are risk-neutral and maximize their respective expected profits. Section 4 studies how risk aversion may change the market outcome and the firms' preferences on information sharing.

\section{Analysis}

We solve the game using backward induction. Section 3.1 characterizes the equilibrium outcomes for the subgames in the second stage (i.e., after the informationsharing format is chosen). Section 3.2 examines different channel members' preferences over the sharing formats. Section 3.3 studies the impact of forecast accuracy on the firms' profits.

\subsection{Subgame Equilibrium Outcome}

The manufacturer's profit is given by $\pi_{M}(w)=w D=$ $w(\theta-p)$. Given the forecast $s$, the manufacturer's expected profit can be written as $E\left[\pi_{M}(w) \mid s\right]=$ $w(E[\theta \mid s]-p)$. Note that after the manufacturer receives the demand forecast, we may view $s$ as the manufacturer's type.

The retailer's profit is $\pi_{R}(p)=(p-w) D=(p-w)$. $(\theta-p)$. If the manufacturer's forecast $s$ is shared with the retailer, her expected profit will be $E\left[\pi_{R}(p) \mid s\right]=$ $(p-w)(E[\theta \mid s]-p)$. If $s$ is not shared, the retailer will make rational inferences about it based on the manufacturer's wholesale price $w$. Manufacturers with different forecasts have different incentives when choosing the optimal $w$. The retailer's expected profit can be expressed as $E\left[\pi_{R}(p) \mid w\right]=(p-w)$. $\left(\sum_{s \in\{l, h\}} \operatorname{Pr}(s \mid w) E[\theta \mid s]-p\right)$. We emphasize that the retailer's inference about $s$ (and ultimately about the market demand) depends on whether no sharing was committed ex ante or voluntarily chosen ex post by the manufacturer. That is, the retailer's rational inference about the forecast under voluntary sharing will be different from that under the no-sharing format. Next we present the subgame equilibrium outcomes under the three sharing formats.
3.1.1. No Information Sharing. Under the nosharing format, the manufacturer ex ante commits to not sharing the forecast with the retailer (e.g., the manufacturer has a strict data confidentiality policy), or the retailer does not make the upfront investment (in technology or human resources) necessary for information sharing. Because the manufacturer chooses its wholesale price $w$ after receiving the forecast, $w$ will reflect its updated belief about demand. Thus, the retailer can make rational inferences about the manufacturer's forecast based on its announced wholesale price. We will use a hat above the variables $\left(^{\wedge}\right)$ to indicate this no-sharing case.

For any given $w$, the manufacturer prefers that the retailer charge a low retail price to induce higher unit sales. However, the retailer's optimal price will be higher if she perceives a higher forecast (about the market size $\theta$ ). Thus, the high-type manufacturer has an incentive to pretend to have received a lower forecast (i.e., mimic a lower-type manufacturer) so that the retailer will set a lower retail price. By contrast, the lowtype manufacturer will have an incentive to separate itself so that the retailer can charge a correspondingly low retail price. To separate itself from the high-type, the low-type manufacturer must distort its wholesale price downward until the high-type manufacturer no longer wants to mimic, i.e., until the high-type manufacturer finds it more profitable to charge a high wholesale price rather than mimicking the low-type manufacturer. The low-type manufacturer weighs the trade-off between the signaling cost, i.e., downward distortion in its wholesale price, and the benefit of separating by using an appropriately low retail price that is in line with the low expected demand. When the market conditions (parameters) are such that the required signaling cost to separate is relatively low, the low-type manufacturer will separate from the high-type. Otherwise, it will give up signaling its low forecast and charge a higher, pooling wholesale price; this gives the manufacturer a higher per-unit profit margin at the expense of lowered unit sales due to the retailer's higher retail price than in the separating case (where she can infer the low-type manufacturer's forecast). From the high-type manufacturer's perspective, the benefit of pooling (mimicking the low-type) is to induce the retailer to set a low retail price to increase unit sales, and the cost of mimicking is a lower profit margin. If the low-type manufacturer's wholesale price is too low, the high-type manufacturer will decide to charge its high, first-best wholesale price (in essence admitting its type) at the expense of reduced unit sales due to the retailer's higher retail price (since her marginal cost is higher and she knows that the demand forecast is high).

Two types of equilibria are possible, i.e., separating and pooling. In the separating equilibrium, the l-type 
manufacturer signals its low forecast by sufficiently reducing its wholesale price (from its first-best level) to prevent profitable mimicking by the $h$-type manufacturer, which will choose its first-best wholesale price. Under pooling, both types of manufacturers choose the same wholesale price. ${ }^{3}$ We refine the multiple equilibria using the lexicographically maximum sequential equilibrium (LMSE) concept (Mailath et al. 1993). In our setting, LMSE refinement selects the unique, most efficient outcome for the $l$-type manufacturer. The intuitive rationale for this refinement is that since the l-type manufacturer wants to reveal its identity, if the l-type manufacturer finds the pooling outcome more profitable than the separating outcome, the pooling outcome should be selected and vice versa. We provide the detailed technical analysis in the online appendix. Let $\tau_{1}$ denote the threshold value on the interval $\left(0, \frac{2}{13}\right)$ that solves $16 \sqrt{\tau_{1}+2 \tau_{1}^{2}}-27 \tau_{1}-2=0$. The following lemma shows the manufacturer's wholesale pricing under the unique LMSE equilibrium.

LEMMA 1. Under no information sharing, a unique LMSE outcome exists, and

(a) If $\rho \Delta \leq \tau_{1}$, the manufacturer will pool at $w=\hat{w}_{1}^{p}=$ $((1-2 \rho \Delta) / 2) \theta$.

(b) If $\tau_{1}<\rho \Delta<1$, the l-type and the h-type manufacturers will separate and choose

$$
w=\hat{w}_{1}^{s}= \begin{cases}\frac{1+3 \rho \Delta-2 \sqrt{\rho \Delta+2 \rho^{2} \Delta^{2}}}{2}, & \text { if } \tau_{1}<\rho \Delta<\frac{1}{2}, \\ \frac{1-\rho \Delta}{2} \bar{\theta}, & \text { if } \frac{1}{2} \leq \rho \Delta<1,\end{cases}
$$

and $w=\hat{w}_{2}^{s}=((1+\rho \Delta) / 2) \bar{\theta}>\hat{w}_{1}^{s}$, respectively.

We use the equilibrium results in Lemma 1 to illustrate the manufacturer's signaling cost and the retailer's strategic pricing behavior. If the l-type manufacturer can share its forecast with the retailer, its optimal wholesale price is $w=((1-\rho \Delta) / 2) \bar{\theta}$. Under the nosharing format, given $\rho \Delta<\frac{1}{2}$, we know $\hat{w}_{1}^{s}=((1+3 \rho \Delta-$ $\left.\left.2 \sqrt{\rho \Delta+2 \rho^{2} \Delta^{2}}\right) / 2\right) \bar{\theta}<((1-\rho \Delta) / 2) \bar{\theta}$, i.e., to signal its type the $l$-type manufacturer must incur a signaling cost by reducing $w$. The retailer's strategic pricing behavior arises because she tends to increase her retail price when she learns the expected demand is higher. When the retailer cannot infer the manufacturer's type, her best-response retail price is $p(w)=\frac{1}{2} \bar{\theta}+w / 2$. By contrast, if the retailer knows that the manufacturer is $h$-type, her best response will be $p(w)=((1+\rho \Delta) / 2) \bar{\theta}+w / 2>$ $\frac{1}{2} \bar{\theta}+w / 2$. That is, the retailer will strategically increase

\footnotetext{
${ }^{3}$ The separating Perfect Bayesian equilibrium (PBE) exists for all parameter values. Yet the pooling PBE exists only when $\rho \Delta \leq \frac{2}{13}$ (i.e., the two types of manufacturers have similar forecasts); otherwise the $h$-type manufacturer can profitably deviate even if it reveals its identity. Our main propositions qualitatively hold the same under pooling and separating outcomes.
}

her retail price when the manufacturer's high demand forecast is known.

Lemma 1 shows that the LMSE outcome is pooling if $\rho \Delta$ is small, and separating if $\rho \Delta$ is large. Note that $E[\theta \mid l]=(1-\rho \Delta) \bar{\theta}$ and $E[\theta \mid h]=(1+\rho \Delta) \bar{\theta}$. Essentially, $\rho \Delta$, the combination of forecast accuracy and forecast variability, is a measure of how much the expected demand varies with the manufacturer's forecast (s). If $\rho \Delta$ is very small, the expected demand changes little regardless of whether the forecast is $h$ or $l$; in this case, as expected, the l-type manufacturer will find it unprofitable to incur the signaling cost to separate. By contrast, if $\rho \Delta$ is large, the expected demand for the $l$-type manufacturer is very different from that for the $h$-type manufacturer, which gives the $l$-type manufacturer more incentive to separate so as to avoid the retailer's setting too high a retail price (which will happen if she believes demand is high).

In equilibrium, the manufacturer's ex ante expected profit (before receiving the forecast) is

$$
\hat{\pi}_{M}= \begin{cases}\frac{1-4 \rho^{2} \Delta^{2}}{8} \bar{\theta}^{2}, & \text { if } 0<\rho \Delta \leq \tau_{1}, \\ \frac{1-2 \rho \Delta-11 \rho^{2} \Delta^{2}+8 \rho \Delta \sqrt{\rho \Delta+2 \rho^{2} \Delta^{2}}}{8} \bar{\theta}^{2} & \text { if } \tau_{1}<\rho \Delta<\frac{1}{2}, \\ \frac{1+\rho^{2} \Delta^{2}}{8} \bar{\theta}^{2}, & \text { if } \frac{1}{2} \leq \rho \Delta<1 .\end{cases}
$$

The retailer's ex ante expected profit is given by

$$
\hat{\pi}_{R}=\left\{\begin{array}{cl}
\frac{1+4 \rho \Delta+4 \rho^{2} \Delta^{2}}{16} \bar{\theta}^{2}, & \text { if } 0<\rho \Delta \leq \tau_{1}, \\
\frac{1-2 \rho \Delta+17 \rho^{2} \Delta^{2}+2(1-5 \rho \Delta) \sqrt{\rho \Delta+2 \rho^{2} \Delta^{2}}}{16} \bar{\theta}^{2}, & \text { if } \tau_{1}<\rho \Delta<\frac{1}{2}, \\
\frac{1+\rho^{2} \Delta^{2}}{16} \bar{\theta}^{2}, & \text { if } \frac{1}{2} \leq \rho \Delta<1 .
\end{array}\right.
$$

3.1.2. Voluntary Information Sharing. We now analyze the voluntary-sharing subgame, where the manufacturer will decide whether to share its forecast $s$ after receiving it (e.g., the manufacturer has a more flexible information-sharing policy). If the manufacturer shares its forecast, the retailer will know s; if the manufacturer does not share, the retailer can make a rational inference on $s$ based on the manufacturer's wholesale price $w$ as well as the fact that it can share but has decided not to. We use a tilde above the variables $(\sim)$ to indicate this voluntary-sharing case.

The l-type manufacturer wants the retailer to know its low forecast so that the retailer will choose a low retail price. Because sharing will costlessly reveal its forecast, clearly the l-type manufacturer will share the 
forecast with the retailer. Hence, the retailer's rational belief is that the manufacturer must be the $h$-type should it decide not to share its forecast. Note that the $h$-type manufacturer is thereby indifferent between sharing and not sharing; either way the retailer will know its type. Thus, for expositional convenience, we assume that the manufacturer will share its forecast if it is indifferent between sharing and not sharing. Therefore, in equilibrium, both types of manufacturers will share the forecast with the retailer and charge the first-best wholesale price. We formally show this result in Lemma 2.

LEMMA 2. Under voluntary information sharing, in equilibrium the manufacturer will always share its forecast with the retailer.

Given the full sharing equilibrium, the manufacturer's and the retailer's expected profits are $\tilde{\pi}_{M}=$ $\left(\left(1+\rho^{2} \Delta^{2}\right) / 8\right) \bar{\theta}^{2}$ and $\tilde{\pi}_{R}=\left(\left(1+\rho^{2} \Delta^{2}\right) / 16\right) \bar{\theta}^{2}$. The full sharing outcome also holds when the forecast has more than two levels or even a continuum of levels. However, $\S 4$ shows that the full sharing outcome may not hold if the retailer is risk-averse; risk aversion can change the retailer's strategic pricing behavior, which reduces the manufacturer's incentive to share its forecast.

3.1.3. Mandatory Information Sharing. Many manufacturers and retailers work closely together to improve channel performance. For example, Nabisco and $P \& G$ develop collaborative planning, forecasting, and replenishment (CPFR) programs with their retailers. In this case, the manufacturer commits to sharing demand information and the retailer determines her retail price based on the shared forecast. Such mandatory sharing may also be a result of the two firms having established long-term relationships or a dominant firm's insistence on forecast sharing. We use a bar above the variables $\left(^{-}\right)$to indicate this mandatory-sharing case. Lemma 2 states that under voluntary sharing, the manufacturer will always share its forecast with the retailer. Thus the equilibrium outcome under mandatory sharing is the same as that under voluntary sharing. The manufacturer's and the retailer's expected profits are $\bar{\pi}_{M}=\left(\left(1+\rho^{2} \Delta^{2}\right) / 8\right) \bar{\theta}^{2}$ and $\bar{\pi}_{R}=\left(\left(1+\rho^{2} \Delta^{2}\right) / 16\right) \bar{\theta}^{2}$.

\subsection{Format Preferences}

Next we analyze the first stage of the game to examine the firms' preferences over the three informationsharing formats. We consider two alternative games based on whether it is the manufacturer or the retailer that makes the sharing format decision since, in practice, both situations may depend on the relative decision power in the channel.

Proposition 1. Voluntary information sharing and mandatory information sharing are equivalent; both firms are indifferent between these sharing formats (i.e., $\tilde{\pi}_{M}=\bar{\pi}_{M}$, $\left.\tilde{\pi}_{R}=\bar{\pi}_{R}\right)$.

The equivalence between voluntary sharing and mandatory sharing for both firms is due to the fact that in equilibrium the manufacturer will always share its forecast under voluntary sharing. For succinctness, we omit the discussion for the voluntary-sharing format here (the results are the same as those in mandatory sharing when no risk aversion is considered).

Proposition 2. If the manufacturer has the decision power to choose the information-sharing format, the equilibrium format will be mandatory information sharing (equivalent to voluntary sharing); if the retailer has the decision power for the information-sharing format, the equilibrium format will be no information sharing. That is, $\hat{\pi}_{M} \leq \bar{\pi}_{M}$ and $\hat{\pi}_{R} \geq \bar{\pi}_{R}$.

One may intuit that the manufacturer will not always want to share its forecast with the retailer while the retailer will always want to know the forecast. However, as Proposition 2 shows, among the three sharing formats, the manufacturer (the informed party) ex ante prefers mandatory sharing (or equivalently, voluntary sharing) whereas the retailer (the uninformed party) prefers no information sharing. The rationale hinges on the low-type manufacturer's signaling cost. Specifically, in the no-sharing case, to induce the retailer to set an appropriately low retail price when the demand forecast is not high, the low-type manufacturer must distort its wholesale price downward to signal its forecast. This signaling cost can be avoided in the mandatory-sharing case. Though the high-type manufacturer will always weakly prefer no sharing, the low-type manufacturer's benefit from avoiding signaling cost is high enough such that ex ante (before learning the demand forecast) the manufacturer prefers mandatory sharing. By contrast, the retailer can significantly benefit from the manufacturer's lower wholesale prices when it signals its low demand forecast; thus the retailer prefers the no-sharing format.

We have assumed that information sharing can be done costlessly. In practice, information-sharing systems may require costly investments by the manufacturer or the retailer. If we model such fixed costs, the firms' sharing preferences may change; for example, if the manufacturer must ex ante incur fixed costs above some threshold to share information later, it may prefer no sharing over any sharing format. Yet when such costs are below the threshold, our results would remain qualitatively the same with similar managerial implications.

\subsection{Forecast Accuracy}

We now examine how forecast accuracy affects the firms' profits under each sharing format.

Proposition 3. Under no information sharing, the manufacturer and the retailer may earn lower profits when 
forecast accuracy improves (i.e., both $\hat{\pi}_{M}$ and $\hat{\pi}_{R}$ may decrease in $\rho$ ); under mandatory or voluntary information sharing, the manufacturer and the retailer will be better off when forecast accuracy improves (i.e., $\tilde{\pi}_{M}, \tilde{\pi}_{R}, \bar{\pi}_{M}$, and $\bar{\pi}_{R}$ all increase in $\rho$ ).

Proposition 3 indicates that under no information sharing, both channel members' profits may decrease in forecast accuracy, i.e., improving forecast accuracy is not necessarily beneficial for either firm. Why can better forecast information hurt both the informed and uninformed parties? The intuition hinges on the impact of forecast accuracy on the firms' strategic pricing behaviors. For the manufacturer, although an accurate forecast reduces demand uncertainty and facilitates the wholesale pricing decision, it can also induce more strategic pricing behavior by the retailer. This gives the high-type manufacturer more incentive to mimic the low-type, hence increasing the manufacturer's signaling cost when the forecast is low. The manufacturer's expected loss from a more accurate forecast due to the retailer's strategic pricing and the signaling cost may outweigh its benefit from the improved wholesale pricing decision, making the manufacturer worse off. For the retailer, although a more accurate forecast may help improve her retail pricing decision, it can also reduce the manufacturer's incentive to separate, reducing the retailer's benefit from the manufacturer's signaling cost (lowered wholesale prices), resulting in lower expected profits for the retailer. This finding is consistent with the well documented "accuracy trap" in the literature (see, e.g., Laucka 2005): An improvement in forecast accuracy does not necessarily lead to increased revenues for the informed party. However, the extant literature assumes that the downstream retailer has superior information whereas in our paper the upstream manufacturer has superior information.

Proposition 3 has useful managerial implications. First, it cautions the manufacturer when making investments in forecasting and market research to obtain better demand information. Conventional wisdom suggests that manufacturers benefit from better demand information (see, for example, the Accurate Response program in Fisher et al. 1994). However, our result suggests that such demand information can be detrimental in a channel without an information-sharing agreement or commitment. Second, the impact of forecast accuracy, and therefore the firms' incentives to improve forecast accuracy, depend on the information-sharing agreement between the channel members. Without information sharing, the firms' and the channel's performances may deteriorate as the manufacturer improves its forecast accuracy. By contrast, under mandatory information sharing, both channel members will benefit from better forecast accuracy; consistent with this result is the fact that $P \& G$ shares its forecasts with retailers and significantly invests each year to improve its demand forecast. Put differently, information sharing creates more incentives for the manufacturer to obtain more accurate forecasts.

\section{Risk Aversion}

Risk aversion is commonplace in business decision making. Unlike national manufacturers, many retailers have smaller scales and may be more risk-averse. Little attention has been paid to risk aversion in the informationsharing literature. We now study the impact of the retailer's risk aversion on the firms' information-sharing preferences by extending our base model to incorporate constant absolute risk aversion in a normally distributed demand framework. To save space, we focus on the qualitatively different results due to the retailer's risk aversion; all detailed model specification and analysis are given in the online appendix.

Our analysis shows that the retailer's risk aversion affects the manufacturer's wholesale pricing and the retailer's retail pricing decisions as well as their sharing preferences. In particular, the impact of having more accurate demand information (i.e., lower uncertainty) is greater on a risk-averse retailer. In other words, a riskaverse retailer will exhibit more strategic retail pricing behavior than a risk-neutral retailer. When the level of demand uncertainty is reduced (via more accurate forecast), a more risk-averse retailer will increase her retail price more than a less risk-averse retailer. This can lead to qualitatively different results compared with the risk-neutral case.

Proposition 4. When the retailer is risk-averse, the manufacturer prefers voluntary sharing to mandatory sharing whereas the retailer prefers mandatory sharing to voluntary sharing.

Proposition 4 shows that, by contrast to the riskneutral case, the manufacturer will prefer voluntary sharing to mandatory sharing when the retailer is risk-averse. This is because information sharing reduces the retailer's demand uncertainty and will lead to a greater increase in retail price from a risk-averse retailer than from a risk-neutral retailer. Thus, even the manufacturer with a low forecast may not always want to share its forecast with the risk-averse retailer. The manufacturer prefers voluntary sharing to mandatory sharing because it can choose not to share its forecast and pool at the same wholesale price when receiving some forecasts; this alleviates the risk-averse retailer's strategic pricing.

Through an extensive numerical study, we find that if the retailer is highly risk-averse, the manufacturer will prefer no sharing to voluntary sharing. That is, even if it has the power in the channel to dictate the information-sharing format, the manufacturer will prefer never sharing its forecast rather than flexibly deciding whether to share after receiving the forecast. 
This result differs from when the retailer is risk-neutral because a more risk-averse retailer will choose a retail price that is significantly lower when demand uncertainty exists, which reduces the manufacturer's incentive to share its forecast. Under the no-sharing format, the manufacturer needs to reduce its wholesale price to credibly convey its low forecast, a signaling cost that can be saved under voluntary sharing. However, under voluntary sharing, in situations where the manufacturer does not share its forecast, the retailer can infer more information about demand (than under the no-sharing format) and more strategically increase her retail price because of reduced demand uncertainty relative to that experienced under the no-sharing format. So, the manufacturer will prefer the no-sharing format when facing a sufficiently risk-averse retailer.

Proposition 4 also shows that, by contrast to the riskneutral case, the risk-averse retailer prefers mandatory sharing to voluntary sharing. Intuitively, this is because mandatory sharing reduces demand uncertainty for the retailer more than voluntary sharing, where the manufacturer may pool and not share its demand forecast in some situations. Our numerical study shows that the retailer will prefer mandatory sharing to no sharing if her risk aversion is sufficiently high. That is, even though under the no-sharing format the retailer can benefit from the manufacturer's signaling cost (a lowered wholesale price) when it wants to convey its low forecast, a strongly risk-averse retailer will suffer more from demand uncertainty that ensues when the manufacturer chooses to pool; as a result, she would prefer mandatory sharing.

Note that if we use the intuitive criterion (Cho and Kreps 1987) rather than LMSE for equilibrium refinement, voluntary sharing and mandatory sharing will be equivalent to both firms. Please see discussions in Section 5.

Proposition 5. If the retailer is strongly risk-averse, higher forecast accuracy may reduce the manufacturer's expected profit under the voluntary-sharing and mandatorysharing formats.

Recall that in the case of a risk-neutral retailer, improving forecast accuracy may hurt the manufacturer only under no information sharing. Proposition 5 shows that the "accuracy trap" can also happen under voluntary sharing and mandatory sharing if the retailer has strong risk aversion. This is again because risk aversion aggravates the retailer's strategic pricing behavior; thus, as forecast accuracy improves, the manufacturer's loss due to the retailer's increased strategic retail pricing behavior can outweigh its benefit from improved wholesale pricing.

Although under mandatory sharing the risk-averse retailer benefits from an improvement in the manufacturer's forecast accuracy, she may be worse off under voluntary sharing, by contrast with the riskneutral case. This is because under voluntary sharing the manufacturer may not share its forecast with the risk-averse retailer; higher forecast accuracy increases the risk-averse retailer's strategic retail pricing behavior, hence increasing the likelihood that the manufacturer will pool to mitigate that behavior, inducing the retailer to lower her retail price. Thus, under voluntary sharing, an improvement in forecast accuracy may hurt the risk-averse retailer as well.

\section{Conclusion and Discussion}

We have examined three information-sharing formats (no sharing, voluntary sharing, and mandatory sharing) in a distribution channel where the manufacturer has superior demand forecast. Our analysis shows several main findings. When the retailer is risk-neutral, voluntary sharing and mandatory sharing are equivalent, and interestingly, the manufacturer prefers mandatory sharing to no sharing and the retailer prefers no sharing to mandatory sharing. Furthermore, a more accurate demand forecast will benefit both firms under voluntary and mandatory sharing, but may lead to lower profits for both firms under no sharing. Under no sharing, an accurate forecast facilitates the manufacturer's wholesale pricing decision, but it also increases the retailer's strategic pricing behavior and the manufacturer's signaling cost, resulting in lower profits for the manufacturer. Moreover, a more accurate forecast helps the retailer to improve her retail pricing decision, but it can make separating less likely, hence reducing the retailer's benefit from the low-type manufacturer's signaling cost (a lowered wholesale price) and resulting in lower expected profits for the retailer. Our finding implies that information sharing in a channel creates more incentives for firms to improve the accuracy of demand forecast.

In a model extension with a risk-averse retailer, we find that voluntary sharing and mandatory sharing are no longer equivalent; instead, the manufacturer prefers voluntary sharing whereas the retailer prefers mandatory sharing. When the retailer is strongly riskaverse, the manufacturer will prefer no sharing to voluntary sharing. By contrast to the risk-neutral case, improving forecast accuracy may lead to lower profits for both firms under voluntary sharing. A common driving force behind these findings is that the riskaverse retailer will strategically raise her retail price to a greater extent than a risk-neutral retailer when she can resolve some uncertainty using better demand forecast. The retailer's risk aversion can therefore significantly influence the manufacturer's incentives for sharing or signaling the forecast. Our research shows that risk aversion plays a critical role in channels and that firms should take this factor into account when making information-sharing and forecast investment decisions. 
This paper has focused on wholesale price contracts. In our model, if the retailer offers the manufacturer a two-part tariff contract, she will simply set the wholesale price at the marginal cost of the manufacturer, who will make zero profit and is indifferent between sharing and not sharing its forecast. In such a case, forecast accuracy does not affect the firm's sharing preferences. By contrast, if the manufacturer offers the retailer a two-part tariff contract, under mandatory sharing the manufacturer's optimal strategy is to set the wholesale price at its marginal cost and charge a lump-sum fee to extract all profits from the retailer (based on the shared forecast). In this case, the manufacturer will be better off as the forecast accuracy increases. Under no sharing, however, the manufacturer with a low forecast will have incentives to mimic a manufacturer with a high forecast by charging the same high lump-sum fee (higher than its own first-best fee). Thus, a separating equilibrium cannot be sustained, the equilibrium will be pooling, and forecast accuracy makes no difference. Under voluntary sharing, a manufacturer with a high forecast will always share its forecast so as not to be mistaken as having a lower forecast. This implies that the equilibrium outcome under voluntary sharing will be the same as that under mandatory sharing. Thus, when the manufacturer specifies the two-part tariff contract, the retailer will make zero expected profit under all three sharing formats. Clearly, the results under two-part tariffs are not interesting and do not reflect reality well.

Profit-sharing contracts are another alternative to wholesale price contracts. In an extension, following Iyer and Villas-Boas (2003), we assume that the manufacturer and the retailer agree to share the total channel profit, a fraction of which will go to the manufacturer and the rest to the retailer. We find that, first, under such profit-sharing contracts, the equilibrium outcome under voluntary sharing is the same as that under mandatory sharing. Second, both the manufacturer and the retailer prefer sharing to no sharing. Third, an improvement in forecast accuracy will benefit both channel members under information sharing, but makes no difference under no sharing. These results are intuitive since sharing forecasts allows the retailer to price better to achieve a higher total channel profit. In short, the results under both two-part tariff and profit-sharing contracts are very intuitive; our analysis of the common wholesale price contracts provides more interesting, qualitatively different results.

We conclude with two caveats. First, we have assumed that under no sharing the manufacturer can ex ante commit to never sharing its forecast. If such commitment is completely not credible, the no-sharing case will reduce to voluntary sharing. If the manufacturer's no-sharing commitment is partial (e.g., no sharing will happen with some probability less than one), the parameter regions for the firms' sharing preferences may change. Second, we have adopted the LMSE concept to refine equilibrium outcomes. An alternative refinement is the intuitive criterion. As is typically the case for static signaling models, the intuitive criterion eliminates pooling equilibria (sometimes separating equilibria as well). In our no-sharing setting, only the separating equilibrium survives the intuitive criterion; under voluntary sharing, only the full-sharing outcome survives. So, with the intuitive criterion refinement, the equilibrium outcomes will be those presented under full separating and full sharing in the respective sharing scenarios. We emphasize, however, that our qualitative results (Propositions 1, 2, 3 , and 5) will continue to hold. This confirms that the main driving forces and intuition in our model (i.e., the manufacturer's signaling cost and the retailer's strategic pricing behavior) are fairly robust. The only exception is Proposition 4; since the intuitive criterion rules out pooling, voluntary sharing and mandatory sharing will be equivalent to both firms. The online appendix provides detailed analysis and discussions of the results under the intuitive criterion.

\section{Supplemental Material}

Supplemental material to this paper is available at http://dx .doi.org/10.1287/mksc.2016.0981.

\section{Acknowledgments}

The authors thank the editor-in-chief, the associate editor, and two anonymous reviewers for helpful comments and suggestions, which have greatly improved this paper. This research is supported in part by the National Natural Science Foundation of China [Grants 71372113 and 71531005]. The authors also thank seminar participants at Washington University in St. Louis and at the 2014 MSOM Conference for helpful comments.

\section{Appendix}

This appendix presents proofs for Propositions 1-3. Other proofs and analysis are in the online appendix.

Proof of Proposition 1. This follows directly from Lemma 2. Also we can derive $\tilde{\pi}_{M}=\bar{\pi}_{M}=\left(\left(1+\rho^{2} \Delta^{2}\right) / 8\right) \bar{\theta}^{2}$ and $\tilde{\pi}_{R}=\bar{\pi}_{R}=\left(\left(1+\rho^{2} \Delta^{2}\right) / 16\right) \bar{\theta}^{2}$.

Proof of Proposition 2. We first consider the manufacturer's preference. When $\rho \Delta \leq \tau_{1}, \hat{\pi}_{M}-\bar{\pi}_{M}=-\left(5 \rho^{2} \Delta^{2} / 8\right) \bar{\theta}^{2}$ $<0$. When $\tau_{1}<\rho \Delta<\frac{1}{2}, \hat{\pi}_{M}-\bar{\pi}_{M}=\left(\left(-\rho \Delta-6 \rho^{2} \Delta^{2}+\right.\right.$ $\left.\left.4 \rho \Delta \sqrt{\rho \Delta+2 \rho^{2} \Delta^{2}}\right) / 4\right) \bar{\theta}^{2}<0$. When $\frac{1}{2} \leq \rho \Delta<1, \hat{\pi}_{M}=\bar{\pi}_{M}=$ $\left(\left(1+\rho^{2} \Delta^{2}\right) / 8\right) \bar{\theta}^{2}$. Thus we know $\hat{\pi}_{M} \leq \bar{\pi}_{M}$. Next we consider the retailer's preference. When $\rho \Delta \leq \tau_{1}, \hat{\pi}_{R}-\bar{\pi}_{R}=$ $\left(\left(4 \rho \Delta+3 \rho^{2} \Delta^{2}\right) / 16\right) \theta^{2}>0$. When $\tau_{1}<\rho \Delta<\frac{1}{2}, \hat{\pi}_{R}-\bar{\pi}_{R}=$ $\left(\left(-\rho \Delta+8 \rho^{2} \Delta^{2}+(1-5 \rho \Delta) \sqrt{\rho \Delta+2 \rho^{2} \Delta^{2}}\right) / 8\right) \bar{\theta}^{2}>0$. When $\frac{1}{2} \leq$ $\rho \Delta<1, \hat{\pi}_{R}=\bar{\pi}_{R}=\left(\left(1+\rho^{2} \Delta^{2}\right) / 16\right) \bar{\theta}^{2}$. Thus we conclude $\hat{\pi}_{R} \geq \bar{\pi}_{R}$.

Proof of Proposition 3. For the manufacturer, when $\rho \Delta \leq$ $\tau_{1}, \hat{\pi}_{M}=\left(\left(1-4 \rho^{2} \Delta^{2}\right) / 8\right) \bar{\theta}^{2}$ and clearly $\partial \hat{\pi}_{M} / \partial \rho<0$. When $\tau_{1}<$ $\rho \Delta<\frac{1}{2}, \partial \hat{\pi}_{M} / \partial \rho=\left(\left(H_{1}-H_{2}\right) /(4 \sqrt{\rho \Delta(1+2 \rho \Delta)})\right) \Delta \bar{\theta}^{2}$, where $H_{1}=6 \rho \Delta+16 \rho^{2} \Delta^{2}>0$ and $H_{2}=(1+11 \rho \Delta) \sqrt{\rho \Delta(1+2 \rho \Delta)}>0$. 
Note $H_{1}^{2}-H_{2}^{2}=\left(14 \rho^{3} \Delta^{3}+27 \rho^{2} \Delta^{2}+12 \rho \Delta-1\right) \rho \Delta$. One can easily show that there exists $\tau_{1}<\rho^{*}<\frac{1}{2}$ such that $\partial \hat{\pi}_{M} / \partial \rho<0$ (i.e., $H_{1}^{2}-H_{2}^{2}<0$ ) when $\tau_{1}<\rho<\rho^{*}$. Differentiating $\tilde{\pi}_{M}$ and $\bar{\pi}_{M}$ yields $\partial \tilde{\pi}_{M} / \partial \rho=\left(\rho \Delta^{2} / 4\right) \bar{\theta}^{2}>0$ and $\partial \bar{\pi}_{M} / \partial \rho=\left(\rho \Delta^{2} / 4\right) \bar{\theta}^{2}>0$. For the retailer, when $\tau_{1}<\rho \Delta<\frac{1}{2}$, $\partial \hat{\pi}_{R} / \partial \rho=\left((34 \rho \Delta-2) \sqrt{\rho \Delta+2 \rho^{2} \Delta^{2}}-\left(40 \rho^{2} \Delta^{2}+11 \rho \Delta-1\right)\right) /$ $\left(16 \sqrt{\rho \Delta+2 \rho^{2} \Delta^{2}}\right) \Delta \bar{\theta}^{2}$; one can readily show that $\partial \hat{\pi}_{R} / \partial \rho<0$ can happen. Differentiating $\tilde{\pi}_{R}$ and $\bar{\pi}_{R}$ gives $\partial \tilde{\pi}_{R} / \partial \rho=$ $\partial \bar{\pi}_{R} / \partial \rho=\left(\rho \Delta^{2} / 8\right) \bar{\theta}^{2}>0$.

\section{References}

Agency Sales (2003) Who provides the marketing information? $33(1): 44-45$

Aviv Y (2001) The effect of collaborative forecast on supply chain performance. Management Sci. 47(10):1326-1343.

Cachon G, Fisher M (2000) Supply chain inventory management and the value of shared information. Management Sci. 46(8):1032-1048.

Cho IK, Kreps D (1987) Signaling games and stable equilibria. Quart. J. Econom. 102(2):179-221.

Chu WJ, Messinger PR (1997) Information and channel profits. J. Retailing 73(4):487-499.

Desai PS (2000) Multiple messages to retain retailers: Signaling new product demand. Marketing Sci. 19(4):381-389.

Desai PS, Srinivasan K (1995) Demand signaling under unobservable effort in franchising: Linear and non-linear price contracts. Management Sci. 41(10):1608-1623.

Dukes A, Gal-Or E, Geylani T (2011) Who benefits from bilateral information exchange in a retail channel? Econom. Lett. 112(2): 210-212.

Fisher ML, Hammond JH, Obermeyer WR, Raman A (1994) Making supply meet demand in an uncertain world. Harvard Bus. Rev. 72(3):83-93.

Gallini NT, Wright BD (1990) Technology transfer under asymmetric information. RAND J. Econom. 21(1):147-160.

Gal-Or E, Geylani T, Dukes A (2008) Information sharing in a channel with partially informed retailers. Marketing Sci. 27(4):642-658.

Guo L (2009) The benefits of downstream information acquisition. Marketing Sci. 28(3):457-471.

Guo L, Iyer G (2010) Information acquisition and sharing in a vertical relationship. Marketing Sci. 29(3):483-506.

Guo X, Jiang B (2016) Signaling through price and quality to consumers with fairness concerns. J. Marketing Res. Forthcoming.

He C, Marklund J, Vossen T (2008) Vertical information sharing in a volatile market. Marketing Sci. 27(3):513-530.
Iyer G, Villas-Boas M (2003) A bargaining theory of distribution channels. J. Marketing Res. 40(1):80-100.

Iyer G, Narasimhan C, Niraj R (2007) Information and inventory in distribution channels. Management Sci. 53(10):1551-1561.

Jiang B, Jerath K, Srinivasan K (2011) Firm strategies in the "Mid Tail" of platform-based retailing. Marketing Sci. 30(5):757-775.

Jiang B, Ni J, Srinivasan K (2014) Signaling through pricing by service providers with social preferences. Marketing Sci. 33(5):641-654.

Jiang B, Sudhir K, Zou T (2016) Cost-information transparency and intertemporal pricing. SSRN Working Paper, Washington University in St. Louis, St. Louis.

Laucka K (2005) The accuracy trap. SDC (July 5), http://www .sdcexec.com/news/10357301/the-accuracy-trap.

Lee HL, So KC, Tang CS (2000) The value of information sharing in a two-level supply chain. Management Sci. 46(5):626-643.

Li L (2002) Information sharing in a supply chain with horizontal competition. Management Sci. 48(9):1196-1212.

Li L, Zhang H (2008) Confidentiality and information sharing in supply chain coordination. Management Sci. 54(8):1467-1481.

Mailath GJ, Okuno-Fujiwara M, Postlewaite A (1993) Belief-based refinements in signaling games. J. Econom. Theory 60(2):241-276.

Miyaoka J, Hausman WH (2008) How improved forecasts can degrade decentralized supply chains. Manufacturing Service Oper. Management 10(3):547-562.

Mohr JJ, Sohi RS (1995) Communication flows in distribution channels: Impact on assessments of communication quality and satisfaction. J. Retailing 71(4):393-416.

Moorthy S, Srinivasan K (1995) Signaling quality with a moneyback guarantee: The role of transaction costs. Marketing Sci. 14(4):442-466.

Shin H, Tunca T (2010) The effect of competition on demand forecast investments and supply chain coordination. Oper. Res. 58(6): $1592-1610$.

Shin J (2005) The role of selling costs in signaling price image. J. Marketing Res. 42(3):302-312.

Simester D (1995) Signalling price image using advertised prices. Marketing Sci. 14(2):166-188.

Taylor TA, Xiao W (2010) Does a manufacturer benefit from selling to a better-forecasting retailer? Management Sci. 56(9):1584-1598.

Tirole J (1988) The Theory of Industrial Organization (MIT Press, Cambridge, MA).

Voluntary Interindustry Commerce Solutions Association (VICSA) (1999) Roadmap to CPFR: The case studies. White paper, Lawrenceville, NJ, http://www.gs1us.org/DesktopModules/ Bring2mind/DMX/Download.aspx?entryid=632. 